Please do not remove this page

RMIT

UNIVERSITY

\title{
Decomposition of evoked potentials using peak detection and the discrete wavelet transform
}

McCooey, Conor; Kumar, Dinesh; Cosic, Irena

https://researchrepository.rmit.edu.au/esploro/outputs/9921859474701341/filesAndLinks?institution=61RMIT_INST\&index=null

McCooey, C., Kumar, D., \& Cosic, I. (2005). Decomposition of evoked potentials using peak detection and the discrete wavelet transform. Proceedings of the 27th Annual International Conference of the IEEE Engineering in Medicine and Biology Society. https://doi.org/10.1109/IEMBS.2005.1616866

Published Version: https://doi.org/10.1109/IEMBS.2005.1616866

Repository homepage: https://researchrepository.rmit.edu.au

(C2005 IEEE. Personal use of this material is permitted. However, permission to reprint/republish this material for advertising or promotional purposes or for creating new collective works for resale or redistribution to servers or lists, or to reuse any copyrighted component of this work in other works must be obtained from the IEEE.

Downloaded On 2023/04/26 23:19:28 +1000 


\title{
Decomposition of Evoked Potentials using Peak Detection and the Discrete Wavelet Transform
}

\author{
Conor McCooey, Student Member, IEEE, Dinesh Kant Kumar, Member, IEEE, \\ and Irena Cosic, Member, IEEE \\ RMIT University \\ PO Box 2476V, \\ Melbourne, VIC 3000, Australia
}

\begin{abstract}
A new method of viewing evoked potential data is described. This method, called the peak detection method, is based on singularity detection using the Discrete Wavelet Transform. The peaks and troughs of raw Visual Evoked Potential data are identified and characterized using the algorithms of this method, resulting in a linear decomposition of the recording into sets of individual peaks. The individual peaks are then added together, averaged and compared to the ensemble average signal. The peak detection method correlates strongly to the ensemble average showing that this method retains the same evoked potential signal profile.
\end{abstract}

Index Terms-Averaging, Discrete Wavelet Transform, EEG, Singularity Detection, Visual Evoked Potentials,.

\section{INTRODUCTION}

Evoked Potentials (EP) are Electroencephalograph (EEG) recordings in response to an external stimulus, where the response is not triggered endogenously by the natural processes of the brain but exogenously by an external trigger to one of the senses. Flash Visual Evoked Potentials (VEP) represent the EEG response to stimulation triggered by a series of flashes presented to a human subject's eye. The EEG response to a single stimulus is not directly observable. The response is masked by other ongoing background EEG events. Typically ensemble averaging of a few hundred epochs of repeated flash stimuli is required to obtain an observable VEP. The background EEG activity, which is assumed to be random in nature, tends towards a zero level as the number of epochs averaged increases. Meanwhile, the stimulus response is treated as time-locked to the stimulus and omnipresent in each epoch and so averages to a particular voltage profile. The latency at which these profile events occur is helpful for clinicians to determine the time delay of the VEP and assess the integrity of the sensory system involved.

From literature, it is evident that there is lack of precise understanding of what exactly EP signals represent. There is no widely acceptable model that explains the details of the generation of this signal. While averaging of EEG is a useful means of extracting some important features of the response, it is known that some information is lost in the process [1].
Reference [2] describes averaged EEG activity as being made up of 3 parts:

“1) a signal which is correlated in time with the stimulus,

2) some signal variations which are less correlated in time with the stimulus, and

3) ongoing activity not related to the stimulus."

The current technique of ensemble averaging has proven efficacy for separating the signal that has temporal correlation (part 1) from the background activity (part 3). However, semi-correlated activity (part 2) may erroneously add to the final VEP depending on its magnitude. EPs are non-stationary and are known to vary from trial to trial [1]. It is very difficult to analyze the spatial origin of EP components because individual events are no longer separable, once averaged.

Furthermore, there has been debate over the origin of EP responses with opinion divided between those who believe it results from stimulus-evoked brain events and those who believe it results from stimulus-induced changes in ongoing brain activity [3]. Finally, EPs require a large number of experiments to be conducted which may not always be appropriate or comfortable for the patient and may itself introduce another source of variation into the VEP due to subject fatigue.

Electroencephalograph (EEG) signals represent a summation of a number of spatially and temporally separated electrical events. Each of these events may be characterized by sharp, irregular non-stationary transitions that are known as singularities. Reference [4] states "Singularities and irregular structures often carry the most important information in signals". Thus, the singularities of EEG recordings may contain useful information regarding the response to the stimulus. This research aims to determine the singularity features of EEG in order toovercome shortcomings of the averaging process. Towards this aim, it is essential that the proposed technique should retain the evoked potential information that is currently available using the ensemble averaging method. This paper tests this by comparing the evoked potential generated using averaging of the singularities and the standard ensemble averaging technique for flash VEP data. The paper also reports analysis of singularities of each of the epochs. 
The authors' previous work [5] has examined the use of modulus maxima singularity detection of the DWT [4] but the shortcoming in the algorithm reported in the paper was that it did not generate a linearly independent set of peaks. This paper reports an enhanced technique where this limitation has been overcome. Here, the singularities are grouped together to identify the individual peaks that would be the basic building block of the EEG recordings. The method is designed to decompose each EP epoch into a linearly independent set of peaks.

The advantage of this decomposition technique is that it allows significantly more flexibility in how averaging may be performed. This is a necessary first step towards an alternative analysis method where selective peaks may be chosen towards the averaging process.

The separation of signals that represent different events can have applications in determining the underlying events that sum up to make the EEG recordings. It is envisaged that this could lead to better understanding of the recordings itself and lend itself to separation of sources and events that generate the EP signals. It may then be possible to identify the response to the stimulus with minimal repetitions.

\section{Peak Detection Method}

This technique requires the detection and identification of peaks in the recordings. As a first step, singularities in the EEG recordings are identified using the DWT based modulus maxima singularity detection method [4]. Expressed using array based logic and analyzed using the peak detection method (PDM) (described in section II B), these singularities are selectively grouped to make a set of discrete peaks that describe the original recording. These linearly independent peaks are recombined for each epoch and compared to the original signal using Pearson's correlation coefficient formula to verify that a close fit is achieved. All peaks across each of the epochs are averaged. This result is compared with the evoked potential generated using the ensemble average method. This comparison is performed to determine whether or not the evoked potential information embedded in the recording is corrupted by the technique. For a better understanding of the technique, a brief of the major concepts is provided below.

\section{A. Wavelets and Singularity Detection}

A wavelet is a time domain wave which is localized, oscillatory and with zero average. A wavelet, $\psi(t)$ may be expressed in terms of a scale or dilation factor, $s$, which when varied compresses or expands the wavelet shape and a translation factor, $u$ that physically shifts the wavelet along the axis (in this case the time axis). The quadratic spline wavelet [4] was used in this research. Wavelets are observed to have similar properties to the peaks we wish to detect insofar as they have large amplitude changes over narrow time changes.

The output of the wavelet transform is a set of coefficients along the time axis for each scale factor [4]. Coefficient values are larger where the signal more closely approximates the particular scaled wavelet at the particular time. Equally, where there is little or no 'fit', coefficient values tend towards zero.

Using a dyadic scale $\left(s=2^{j}\right.$ where $\left.j=1,2,3, \ldots\right)$, a practical implementation of the DWT using a filter bank algorithm called the "algorithme à trous" may be implemented [6]. It generates a detail component (similar to a low pass filter) at each $2^{j}$ scale up to the maximum calculated scale, together with a remaining approximation component.

By selecting a mother wavelet function that is the derivative of a smoothing function, such as the quadratic spline wavelet of degree 2, Reference [4] proved that by selecting the modulus maxima DWT coefficients that had a certain evolution across scales, one could detect local singularities and in the process, demonstrated that a large set of wavelet coefficients could be simplified into a much smaller discrete set of coefficients grouped per singularity. This is a powerful step forward in the characterization of a signal into a group of singularities. An inverse discrete wavelet transform using the selected singularity coefficients was demonstrated to approximate the original signal well.

\section{B. Peak Detection}

Singularity detection using the discrete wavelet transform (DWT) described by Reference [4] is the starting point for the theory outlined in this paper. Singularities characterize areas where large magnitude change in the signal over a short period occurs i.e. points where there is a large slope. A peak may be considered to be a set of two or three singularities that record a simple shift away from and back to a particular baseline. The position and value of both the detail and approximation components are recorded in a single line of an array which defines the singularity. The singularities are matched into pair or triplets that identify distinct peaks within the data. Sets of singularities are then identified that combine to form peaks using the following set of rules:

i. A singularity characterizing a bi-phasic transition is split into two singularities - one characterizing the positive transition and the other the negative transition. This allows a bi-phasic peak to be divided into a pair of mono-phasic peaks.

ii. A pair of singularities is more likely to form a peak if the absolute magnitudes of the detail components at the highest scale level for each singularity are similar to one another.

iii. Singularities that increase with time have positive coefficients and those that decrease with time have negative coefficients. A peak must have both.

iv. Singularities that match to form a peak should have similar maximum detail scale level $(s)$ within \pm 1 scale factor of each other.

v. The gap between the position of the first detail component of each singularity in a pair is related to the dyadic scale factor fit the following rule: 


$$
2^{s j}<\operatorname{pos}_{1}\left(s_{2}\right)-\operatorname{pos}_{1}\left(s_{1}\right)<2^{s+1}
$$

where $\operatorname{pos}_{I}\left(S_{x}\right)$ represents the position of the first coefficient value for singularity $\left(s_{x}\right)$ (pos 1 in Table 1$)$

The PDM algorithm matches the array of singularities using these rules to yield a single peak. The process is then repeated for all remaining singularities in the epoch until all singularities are matched into a set of linearly independent peaks. Inverting (inverse DWT) and adding together these peaks yields a close approximation of the original signal.

\section{RESULTS}

\section{A. Experimental Setup}

Ethics approval for the experimental procedure was obtained from the local human research ethics committee. Flash Visual Evoked Potential signals were recorded for a single subject. Repeated strobe light flashes were presented to the subject's visual field at a distance of about 1 meter from the eye at an approximate rate of 1 flash per second. The EEG response was measured from the MidOccipital(MO) position, which is located $5 \mathrm{~cm}$ directly above the inion [7]. An epoch length of 500ms was recorded synchronously with the strobe flash where the flash occurred at 0 seconds in each epoch. The sample rate was 512 per second. Data was normalized to give a zero mean across all data. Partial peaks occurring at the edges of each epoch were removed to eliminate border effects from the subsequent DWT processing. Visual inspection was performed to remove gross artifacts. A total of 104 epochs were initially used for analysis.

\section{B. Preliminary Results}

For a better understanding to the peak detection process, an example consisting of analysis of a representative peak is given in Fig 1. The original signal is a small section of the recording extracted, for illustrative purposes, using a temporal window of size $25 \mathrm{~ms}$ and corresponding to a single peak. The modulus maxima DWT coefficients of this signal are shown in Table 1 as Sing1 and Sing2. Sing1 characterizes the upward slope of the original signal at 20-30ms and Sing2 characterizes the downward slope of the original signal at $35-45 \mathrm{~ms}$. The peak has been characterized by considering both, Sing1 and Sing2 and an inverse DWT is performed, resulting in the reconstructed signal shown as the PDM signal in Fig. 1.

The DWT generates coefficients that are made up of detail and approximation components. In Table 1, the $l v l$ column indicates the max scale used to characterize singularities or peaks. A $l v l$ of 4 indicates all detail coefficients from 1 to 4 are used to characterize the peak. The coefficients, $A_{l v l}, A_{p}$, $A_{a}$, give the correct approximation information in terms of

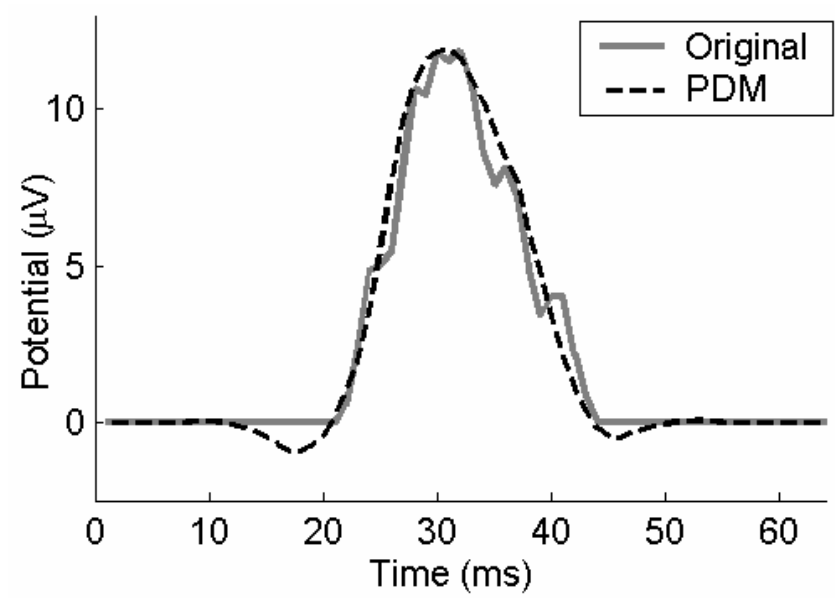

Fig 1. An example of how the Peak Detection Method signal (PDM) approximates an EEG signal (Original).

level, coefficient position and coefficient magnitude. $A_{l v l}=0$ means that this approximation coefficient is not used to regenerate the peak.

\section{Epoch Results}

The PDM was applied to each epoch to identify and characterize the major peaks. The results from each epoch were manually inspected and occasional scale ( $l v l)$ adjustment was required to ensure accurate peak reconstruction. This process shall be automated in future research into this method.

Pearson's correlation coefficient, $r$, was calculated for each epoch signal, $A$ and its corresponding PDM signal, $B$ using:

$$
r=\frac{\sum_{n}\left(A_{n}-\bar{A}\right)\left(B_{n}-\bar{B}\right)}{\sqrt{\left(\sum_{n}\left(A_{n}-\bar{A}\right)^{2}\right)\left(\sum_{n}\left(B_{n}-\bar{B}\right)^{2}\right)}}
$$

where $\bar{A}, \bar{B}$ represent signal means and $n$ is the number of data points.

Fig 2. illustrates a histogram of correlation coefficients for all 104 epochs. A correlation coefficient of 1 represents a perfect match between signals. Three epochs were classified as outliers with correlation coefficients less than 0.86 and were removed from further analysis leaving 101 epochs from a total of 104. The mean correlation coefficient of these is 0.9560 with a standard deviation of 0.0211 .

\section{Averaging Results}

The standard ensemble average and the PDM average were calculated for the 101 epochs and the results are shown in Fig 3. It is noted that the PDM average gives an excellent approximation of the ensemble average signal with a correlation coefficient of 0.9948, indicating that decomposing the evoked potential response into a set of

TABLE 1

\begin{tabular}{|c|c|c|c|c|c|c|c|c|c|c|c|c|c|c|}
\hline Sing & $l v l$ & $\operatorname{pos}_{1}$ & $\operatorname{det}_{1}$ & $\operatorname{pos}_{2}$ & $\operatorname{det}_{2}$ & $p^{\prime} s_{3}$ & $\mathrm{det}_{3}$ & $p^{2} s_{4}$ & $\operatorname{det}_{4}$ & $p^{2} s_{5}$ & $\operatorname{det}_{5}$ & $A_{l v l}$ & $\overline{A_{p}}$ & $A_{a}$ \\
\hline 1 & 4 & 22 & 1.7 & 21 & 3.0 & 21 & 7.4 & 16 & 12.8 & 0 & 0 & 0 & 14 & 0.4 \\
\hline 2 & 4 & 36 & -1.6 & 35 & -2.8 & 32 & -5.3 & 30 & -11.5 & 0 & 0 & 4 & 39 & 24.0 \\
\hline
\end{tabular}

COEFFICIENT SETS OR SINGULARITIES FOR THE PEAK SHOWN IN FIG 1. DETERMINED BY THE PEAK DETECTION METHOD. 
peaks has retained the information that is observable by ensemble averaging.

\section{CONCLUSION}

The peak detection method is described and tested for flash VEP data taken from a single subject. The results indicate a high level of correlation between the signals generated using PDM and the original EEG recording. Excellent correlation is also demonstrated when comparing the results with evoked potential generated using ensemble averaging, demonstrating that this technique gives results that are not inferior to the conventional averaging process.

The PDM may be used to characterize EP data into a set of linearly independent peaks that closely approximate the original signal. Applications for this method are not described herein. The next step is to develop a classification mechanism to identify peaks more likely to be associated with EP signals and less likely to be background EEG. This is the topic of further study by the authors.

\section{REFERENCES}

[1] J. Zhang, C. Zheng, "Extracting Evoked Potentials with the Singularity Detection Technique", IEEE Engineering in Medicine and Biology, pp 155-161, Sept. 1997.

[2] C. Kooi, "Visual evoked potentials in central disorders of the visual system," Harper \& Row, $1^{\text {st }}$ edition, 1979.

[3] S. Makeig, M. Westerfield, T.-P. Jung, S. Enghoff, J. Townsend, E. Courchesne, T. J. Sejnowski, "Dynamic Brain Sources of Visual Evoked Responses," Science, vol. 295, pp. 690-694, 2002.

[4] S. G. Mallat, W. L. Hwang, "Singularity detection and processing with wavelets," IEEE Trans. on Info. Th., vol. 38, pp. 617-643, March 1992.

[5] C. McCooey, D. Kant Kumar, "Characterising evoked potential signals using wavelet transform singularity detection", World Congress on Medical Physics and Biomedical Engineering, Sydney, 2003.

[6] S. G. Mallet, "A Wavelet Tour of Signal Processing", Second Edition, Academic Press, 1999.

[7] K. E. Misulis, "Spehlmann's Evoked Potential Primer", Second Edition, Butterworth-Heinemann, 1994.

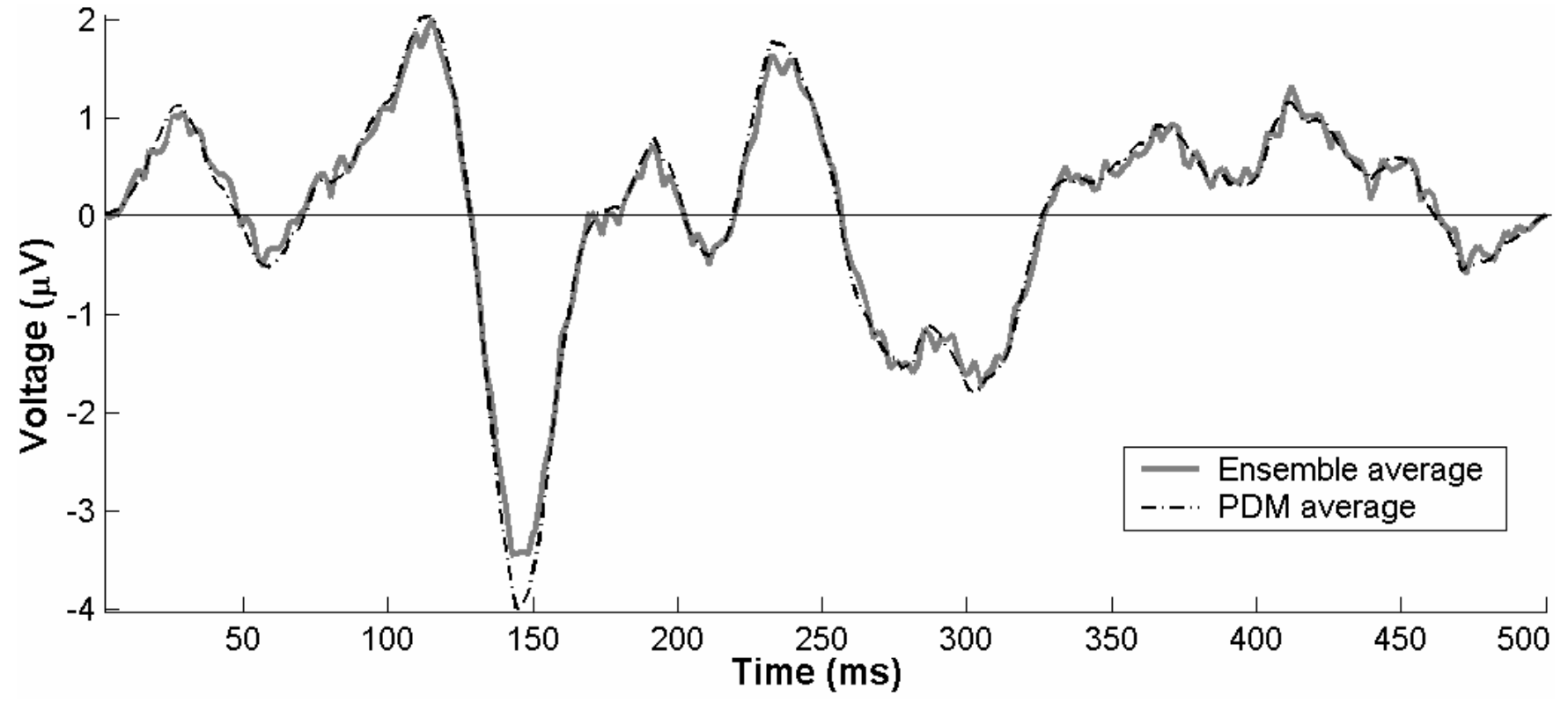

Fig 3. Comparison of standard ensemble average VEP and average of peaks detected for all epochs using the PDM.

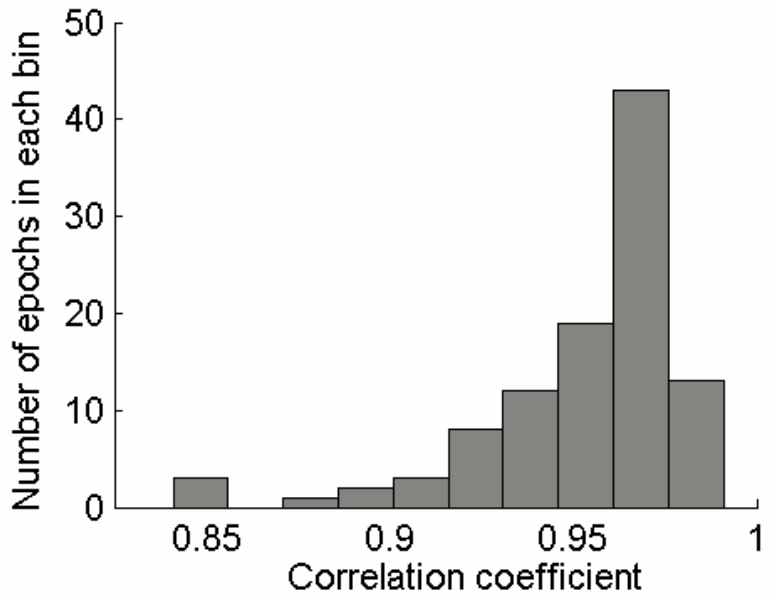

Fig 2. Correlation coefficients for 104 epochs of data. The three epochs below 0.86 were removed leaving 101 epochs. 